\title{
Estudio Comparativo de Embarazadas Adolescentes versus Embarazadas Adultas Jóvenes en el Centro Hospital Primitivo Iglesias de la Ciudad de Cali
}

\author{
Dres.: María Clara Tovar*, Consuelo Burbano**, Martha Patricia Mora***, \\ Martha Lucía Domínguez****, Ana Helena García****.
}

\section{EXTRACTO}

Se realizó un estudio longitudinal comparando un grupo de embarazadas primigestantes menores de 18 años y otro de primigestantes adultas jóvenes entre 20 y 29 años $y$ en ambos grupos se analizaron algunos aspectos médicos y sociales.

El estudio se llevó a cabo en el Centro Hospital Primitivo Iglesias de la Ciudad de Cali, Centro dentinado a la atención del bajo riesgo.

En las gestantes adolescentes se presentó con más frecuencia el parto prematuro, el abandono de los estudios y el rechazo

Enf. Obst. MSP. Profesor Auxiliar

Departamento Enfermería Univalle.

** Estudiante Enfermería Univalle

*** Estudiante Enfermería Univalle

**** Estudiante Enfermería Univalle

****** Estudiante Enfermería Univalle familiar aunque en éste último las diferencias no fueron estadísticamente significativas, en contraste con la mujer adul ta joven donde fue más frecuente el abandono del trabajo.

Agradecemos a la doctora Graciela Pardo de Vélez por la asesoría en el trabajo y al ingeniero Héctor Angulo del Centro de Cómputo de la Universidad del Valle por el procesamiento de la información.

\section{INTRODUCCION}

En Colombia y en el mundo existe actualmente una justificada preocupación por un sector crítico de la población en el cual descansa su futuro inmediato, el sector de la población adolescente.

Cali la capital del Valle no se escapa a ésta situación si tenemos en cuenta que para 1986 el 25 por ciento de ingresos a la sala de partos del Centro Hospital Pri- 
mitivo Iglesias, lo constituyeron gestantes menores de 18 años.

El estudio pretende identificar alguras complicaciones médicas y sociales presentadas en madres adolescentes comparadas con madres adultas jóvenes tales como: Hipertensión inducida por el embarazo, parto prematuro, madre-solterismo, rechazo de la familia por el embarazo, abandono del estudio $y / 0$ trabajo e intento de aborto y de suicidio.

El embarazo en adolescentes constituye un problema de salud pública puesto que contribuye a aumentar la morbi-mortalidad materna e infantil (1). En la región de las Américas este factor ha sido considerado como problema prevalente, objeto de especial preocupación, desde comienzos del decenio de 1980 (2).

El desarrollo general insuficiente, la falta de equilibrio del sistema endocrino, la tensión emocional en el primer embarazo, la dieta insuficiente, hacen que se presenten problemas tanto médicos como sociales relacionados a la obstetricia del adolescente.

En adolescentes gestantes solteras la situación se agrava porque afrontan problemas síquicos y sociales sobreagregados a aquellos de índole puramente orgánico como: abandono de los estudios, búsqueda del sustento diario, dependencia de su familia, intento de aborto y de suicidio. (3) La problemática se hace crítica debido a las características de la salud materno infantil en la América Latina, en donde las elevadas tasas de mortalidad infantil, perinatal y materna están asociadas a deficiencias, tanto en las condiciones de vida como en los recursos y organización de los servicios de atención médica (4).

El porcentaje de adolescentes en embarazo varía mucho en las diferentes regio- nes del mundo: según Zuckerman, S. Barry y colaboradores (5), en una revisión bibliográfica de las consecuencias del embarazo en la adolescencia en los Estados Unidos menciona cómo va aumentando el embarazo en éste grupo de edad y es así como las tasas de nacimientos para adolescentes de 10 a 14 años ha aumentado entre 1966 y 1981 un 22 porciento mientras que para el grupo de 15 a 19 años ha disminuido. Vale la pena anotar que los adolescentes entre los 15 y 19 años son cerca del 10 por ciento del total de la población mundial.

En la república de Corea las adolescentes entre 15 y 19 años son responsables del 2 por ciento de los nacimientos $y$ en Cuba del 23 por ciento con un total en el mundo de 13 millones de nacimientos de madres adolescentes y cada año 1 de cada 10 nacimientos corresponde a madres adolescentes (6).

En Jamaica (7) tradicionalmente la mujer comienza su maternidad en edad temprana, pero en los últimos años ha aumentado la tasa de natalidad en adolescentes.

En 1973 en el hospital más grande de Kingston los partos de adolescentes representaron un 26 por ciento del total y para 1976 esta cifra se había elevado al 33 por ciento.

En Colombia según Alberto Rizo y $\mathrm{He}$ lena Prada (8) la población adolescente es casi la tercera parte del total de la población en la actualidad.

Horión y colaboradores (9) en 1983 al comparar un grupo de niños nacidos de primigrávidas adolescentes menores de 16 años con un grupo de niños hijos de madres de 20 a 24 años, halló menor edad gestacional en los niños de adolescentes. Las madres adolescentes tenían más bajo 
peso antes del embárazo, menos estatura, e iniciaron el control prenatal más tarde; el 88.6 por ciento de las adolescentes eran madres solteras, todos estos factores contribuyeron a aumentar el riesgo.

Roosevelt C. (10) en 1986 en Campinas (Brasil) en un trabajo sobre suicidio en adolescencia y tendencias sicosociales menciona cómo un $8 \%$ de esos suicidios son debidos a una gravidez indeseada.

Zuckerman S. Barry y colaboradores (11) en 1984, describen cómo desde 1922 muchos investigadores hail notado la asociación de embarazo en adolescentes y las complicaciones obstétricas y morbi-mortalidad neonatal.

Las adolescentes frecuentemente buscan el aborto o intento de aborto como solución al embarazo.

Belitzky, Rubén, Campo C. Aníbal y colaboradores con datos de 17 maternidades latinoamericanas provenientes de 10 países de la región todas consideradas de nivel terciario en su medio y cuyos resultados no son representativos de países, muestra la frecuencia de madres adolescentes y cómo varía según el límite de edad que se emplee para la clasificación.

En el estudio incluye el Hospital Universitario del Valle con un porcentaje de 18.8 por ciento de nacimientos en menores de 18 años, cifra alta si se compara con tasas de otros países, como también los riesgos de éste grupo de edad.

La mortalidad perinatal en el Hospital Universitario del Valle en el grupo de adolescentes comparada con el grupo de 20 a 24 años casi que se duplica siendo de 72.7 por ciento y 44.6 por ciento respectivamente.
En general, los indicadores perinatales reflejan peores resultados en las madres más jóvenes que en las de edad subsiguiente.

Señala el grupo más joven como de mayor riesgo. Por la frecuencia de resultados adversos a corto y largo plazo y la posibilidad de influir favorablemente con acciones médico-sociales, éste grupo merece una organización especial antes, durante $y$ después del embarazo.

\section{METODO Y DISEÑO}

El Municipio de Cali para la atención en salud se halla dividido en 4 áreas básicas con un centro hospital como cabecera de área.

El esstudio se realizó en el Centro Hospital Primitivo Iglesias (CHPI) cabecera del área No. 3 y del cual dependen 5 puestos y 4 centros de salud con una población de 340.668 habitantes para 1986 según proyecciones de población de acuerdo al censo de 1973, con una población asignada del 60 por ciento.

El Centro Hospital posee los servicios básicos de medicina interna, obstetricia, pediatría y cirugía, prestando atención a personas de bajo y mediano riesgo relacionados con las 4 áreas básicas. La atención terciaria se remite al Hospital Universitario del Valle.

La población obstétrica para 1986 fue de 8.889 gestantes. Para 1985 el CHPI atendió 2.141 partos de los cuales el 25 por ciento correspondieron a gestantes menores de 19 años.

El Universo lo constituyó las embarazadas que asistieron a la atención del parto al Centro Hospital Primitivo Iglesias, en los meses de octubre de 1986 a febrero de 1987 . 
Para la muestra se tomaron las embarazadas adolescentes que llegaron a la sala de partos durante los meses ya mencionados.

Por cada adolescente se seleccionó 1 adulta joven que ingresó a la sala en la misma semana. Se igualaron los 2 grupos respecto a primigravidez y nivel socioeconómico. Para la recolección de la información se diseñó un formulario de 15 preguntas de las cuales 8 preguntas eran dicotómicas y 7 de escogencia múl tiple aplicado a ambos grupos. El formulario fue probado con gestantes de la sala de puerperio del Hospital Universitario del Valle al cual se le realizaron sus respectivos ajustes después de la prueba.

El estado civil se discriminó en ambos grupos según las siguientes categorías: solteras, cuando no tenían pareja estable, unión libre cuando existía una unión estable mayor de un año y casadas cuando había pareja estable y el vínculo se había formalizado con matrimonio civil o católico.

Para el análisis de la información se realizaron cuadros y se aplicó el Chi cuadrado para calcular la asociación entre cada una de las variables estudiadas. Se calculó la tasa de incidencia de factores médicos y sociales para ambos grupos lo que permitió calcular el riesgo relativo, el cual es una comparación de la frecuencia con que ocurre el daño en los individuos que tienen el atributo o factor de riesgo y la frecuencia con que acontece en aquellos que no tienen el factor de riesgo (13).

Tasa de incidencia de expuestos al factor (es) de riesgo

$\mathrm{R} R$

Tasa de incidencia de no expuestos al factor (es) de riesgo

\section{RESULTADOS}

El estudio comparativo entre gestantes adolescentes y embarazadas adultas jóvenes realizado en el Centro Hospital Primitivo Iglesias, proporciona información para ambos grupos acerca del estado civil, intento de aborto, intento de suicidio, rechazo de la familia, bajo peso al nacer, ruptura prematura de membranas e hipertensión inducida por el embarazo.

Se encontró una proporción mayor de madres adolescentes solteras. Aunque los datos de madres solteras en el grupo de adolescentes es menor al encontrado por Duarte Contreras en 1960 - 1971 donde halló que el 70.8 por ciento de gestantes adolescentes solteras, el dato en este estudio de 37 por ciento de adolescentes solteras supera a las gestantes de 20 a 29 años, 26 por ciento (ver Cuadro No. 1).

Respecto a la categoría casadas la proporción es mayor 26 por ciento en el grupo de adultas jóvenes contrastando con un 8 por ciento en el de adolescentes.

Para unión libre no hay una mayor diferencia entre ambos grupos lo cual está de acuerdo con las características del área donde se realizó el estudio,pues el patrón de la población es la unión libre.

El Cuadro No. 2 muestra los resul tados obtenidos en cuanto a las variables sociales incluidas en el estudio.

A la pregunta "si habían pensado o intentado quitarse la vida en algún momento a causa de su embarazo"; el intento de suicidio mostró un 4 por ciento para las adolescentes embarazadas, porcentaje bajo comparado con el reportado por Gabrielson (14) en un estudio llevado a cabo en Campinas (Brasil) en 1970 donde muestra un 22 por ciento de intento de suicidio en madres solteras adolescentes. 
CUADRO No. 1

DISTRIBUCION DEL ESTADO CIVIL EN ADOLESCENTES

EMBARAZADAS Y ADULTAS JOVENES ATENDIDAS

EN EL CHPI - CALI

OCTUBRE 1986 - . FEBRERO 1987

\begin{tabular}{|c|c|c|c|c|}
\hline \multirow[t]{2}{*}{ ESTADO CIVIL } & \multicolumn{2}{|c|}{$\begin{array}{l}\text { EMBARAZADAS } \\
\text { ADOLESCENTES }\end{array}$} & \multicolumn{2}{|c|}{$\begin{array}{c}\text { EMBARAZADAS } \\
\text { ADULTAS JOVENES }\end{array}$} \\
\hline & No. & $\%$ & No. & $\%$ \\
\hline Solteras & 18 & 37.0 & 13 & 26.0 \\
\hline Unión Libre & 2.7 & 55.0 & 24 & 48.0 \\
\hline Casadas & 4 & 8.0 & 13 & 26.0 \\
\hline TOTAL & 49 & 100. & 50 & 100. \\
\hline
\end{tabular}

CUADRO No. 2

INCIDENCIA DE COMPLICACIONES SOCIALES EN EMBARAZADAS ADOLESCENTES COMPARADAS CON EMBARAZADAS ADULTAS JOVENES

\begin{tabular}{|c|c|c|c|c|c|}
\hline \multirow[t]{2}{*}{$\begin{array}{l}\text { COMPLICACIONES } \\
\text { SOCIALES }\end{array}$} & \multicolumn{2}{|c|}{$\begin{array}{l}\text { EMBARAZADAS } \\
\text { ADOLESCENTES }\end{array}$} & \multicolumn{3}{|c|}{$\begin{array}{c}\text { EMBARAZADAS } \\
\text { ADULTAS JOVENES }\end{array}$} \\
\hline & No. & $\%$ & No. & $\%$ & $\mathrm{RR}$ \\
\hline Intento de suicidio & 2 & 4 & 1 & 2 & 2 . \\
\hline Intento de aborto & 11 & 22. & 3 & 6 & 3.66 \\
\hline $\begin{array}{l}\text { Rechazo de la fa- } \\
\text { milia. }\end{array}$ & 17 & 35 & 9 & 18 & 1.94 \\
\hline Abandono del trabajo & 4 & 8 & 17 & 34 & 0.23 \\
\hline Abandono del estudio & 15 & 31 & 2 & 4 & 7.75 \\
\hline
\end{tabular}


El porcentaje de intento de aborto es de un 22 por ciento contrastando con un 6 por ciento en las adultas jóvenes, esto sin tener en cuenta las que verdaderamente abortan al inicio del embarazo.

Respecto al rechazo de la familia el grupo de menor edad es el que se ve más afec. tado. (Ver Cuadro No. 2).

Los resultados del abandono de los estudios son inversos a los resultados del abandono del trabajo. En el grupo de adolescentes abandonó el estudio el 31 por ciento de las gestantes contrastando con sólo un 2 por ciento en las adultas jóvenes.

El abandono del trabajo fue más elevado $(34 \%)$ en las madres adultas jóvenes que en el grupo de menor edad (4\%). (Cuadro No. 2).

Este último resultado sugiere que el grupo de adolescentes estudia en mayor número y por lo tanto, el abandono del estudio muestra un alto porcentaje de esta población, lo contrario ocurriría con el grupo de mayor edad que trabaja más y por lo tanto abandona en mayor proporción su trabajo.

El presente estudio nos permitió obtener datos sobre la incidencia de las complicaciones médicas de madres y recién nacidos; en los grupos se incluyó bajo peso al nacer, ruptura prematura de membranas, parto prematuro e hipertensión arterial inducida por el embarazo.

Para identificar el bajo peso al nacer se tomó el dato del libro de registro de parto, definiendo como de bajo peso al recién nacido mayor de 37 semanas de gestación y con un peso menor de 2.500 gramos.

El estudio mostró un 10 por ciento de bajo peso al nacer para el grupo de adolescentes mientras que para el otro grupo no se registró ninguno. Igualmente sucedió con la ruptura prematura de membranas, el parto prematuro y la hipertensión arterial inducida por el embarazo, con cifras mayores para el grupo de adolescentes comparado con el otro grupo. (Cuadro No. 3).

CUADRO No. 3

\section{PORCENTAJE DE COMPLICACIONES MEDICAS DE MADRES $Y$ RECIEN NACIDOS EN EMBARAZADAS ADOLESCENTES $Y$ EMBARAZADAS ADULTAS JOVENES}

\begin{tabular}{|c|c|c|c|c|c|}
\hline \multirow[t]{2}{*}{$\begin{array}{c}\text { COMPLICACIONES } \\
\text { MEDICAS }\end{array}$} & \multicolumn{2}{|c|}{$\begin{array}{l}\text { EMBARAZADAS } \\
\text { ADOLESCENIES }\end{array}$} & \multicolumn{2}{|c|}{$\begin{array}{l}\text { EMBARAZADAS } \\
\text { ADULTAS JOVENES }\end{array}$} & \multirow[b]{2}{*}{ R R } \\
\hline & No. & 8 & No. & $\%$ & \\
\hline Bajo peso al nacer & 5 & 10 & 0 & - & \\
\hline $\begin{array}{l}\text { Ruptura prematura } \\
\text { de membranas }\end{array}$ & 7 & 14 & 4 & 8 & 1.75 \\
\hline Parto prematuro & 23 & 47 & 5 & 10 & 4.7 \\
\hline Toxemia & 2 & 4 & 3 & 6 & 0.6 \\
\hline
\end{tabular}


Respecto al riesgo relativo el más alto se presenta para parto prematuro mostrando el grupo de menor edad con 4.7 veces más riesgo de tener un parto prematuro que el grupo de 20-29 años (Cuadro No. 3).
Las diferencias son estadísticamente significativas para parto prematuro, lo cual muestra aspectos en los cuales puede intervenir el equipo de salud con una mayor vigilancia para este grupo de población (Cuadro No. 4).

\section{CUADRO No. 4}

\section{FRECUENCIA DEL PARTO PREMATURO EN EMBARAZADAS ADOLESCENTES Y ADULTAS JOVENES}

\begin{tabular}{|lcccc|}
\hline & $\frac{\text { PARTO }}{\text { SI }}$ & $\frac{\text { PREMATURO }}{\text { NO }}$ & TOTAL \\
\hline Embarazada adolescente & 23 & & 27 & 50 \\
Embarazada adulta joven & 5 & & 44 & 49 \\
T O T A L & $-\frac{71}{28}$ & & & 99 \\
\hline
\end{tabular}

Chi $^{2}=15.63 \quad \mathrm{GL}=1 \quad \mathrm{P}=<0.05$

CUADRO No. 5

FRECUENCIA DE ABANDONO DEL ESTUDIO SEGUN EMBARAZADAS ADOLESCENTES Y ADULTAS JOVENES

\begin{tabular}{|lcccc|}
\hline & \multicolumn{3}{c|}{ ABANDONO DEL ESTUDIO } \\
& $-\frac{\text { SI }}{\text { No }}$ & & No & TOTAL \\
\hline Embarazada adolescente & 15 & 30 & No. \\
Embarazada adulta joven & -2 & & 47 & 45 \\
Total & 17 & & 77 & 49 \\
\hline
\end{tabular}

$\mathrm{Chi}^{2}=13,54 \quad \mathrm{GL}=1 \quad \mathrm{P} \doteq<0.05$ 
El abandono del estudio mostró diferencias significativas para el grupo de adolescentes (Cuadro No. 5) mientras que el abandono del trabajo mostró diferencias significativas para el grupo de adultas jóvenes (Cuadro No. 6).

CUADRO No. 6

FRECUENCIA DE ABANDONO DEL TRABAJO SEGUN EMBARAZADAS ADOLESCENTES Y ADULTAS JOVENES

\begin{tabular}{|c|c|c|c|}
\hline & \multicolumn{3}{|c|}{ ABANDONO DEL TRABAJO } \\
\hline & SI & NO & TOTAL \\
\hline & No. & No. & No. \\
\hline & & & \\
\hline Embarazada adolescente & 4 & 43 & 47 \\
\hline Embarazada adulta joven & 17 & 32 & 49 \\
\hline TOTAL & 21 & 75 & 96 \\
\hline
\end{tabular}

Chi $^{2}=8.15 \quad \mathrm{GL}=1 \quad \mathrm{P}=<0.05$

CUADRO No. 7

FRECUENCIA DEL RECHAZO FAMILIAR EN EMBARAZADAS ADOLESCENTES $Y$ EMBARAZADAS ADULTAS JOVENES

\begin{tabular}{|c|c|c|c|}
\hline & \multicolumn{3}{|c|}{ RECHAZO FAMILIAR } \\
\hline & SI & NO & TOTAL \\
\hline $\begin{array}{l}\text { Embarazadas adoles- } \\
\text { centes. }\end{array}$ & 17 & 33 & 50 \\
\hline $\begin{array}{l}\text { Embarazadas adultas } \\
\text { jóvenes. }\end{array}$ & 9 & 41 & 50 \\
\hline TOTAI & 26 & $74^{\prime}$ & 100 \\
\hline
\end{tabular}

$\mathrm{Chi}^{2}=.3 .3$

$\mathrm{GL}=1$

$P>0.5$ 


\section{DISCUSION Y CONCLUSIONES}

La adolescencia, biológica y socialmente no es un problema; es un proceso normal de desarrollo y maduraciônn. Una serie de situaciones resultantes del ambiente en que se desenvuelve, llevan a las adolescentes a exponerse a riesgos como el embarazo.

Los organismos e instituciones públicas y privadas deberán aceptar el reto ante el diagnóstico de una realidad preocupante, aplicando las medidas que se dirijan a corregir, proteger y prevenir a nuestra juventud de todos aquellos riesgos $y$ peligros que representa una maternidad precoz.

El estudio muestra una mayor proporción de problemas sociales y médicos para la joven adolescente, aspecto a tener en cuenta cn la educación a los grupos de jóvenes recalcando los riesgos y la responsabilidad en la sexualidad y la maternidad.

En busca de una verdadera integración docente asistencial los sectores educación y salud pueden trabajar coordinadamente con el adolescente proporcionándole cducación y salud. El embarazo en adolescentes no es sólo responsabilidad de la mujer ya que para ello fue necesario un hombre, quien también debe ser educado conjuntamente para que asuma su sexualidad con responsabilidad.

Es importante realizar investigaciones de cohortes para medir algunos riesgos que se descubren durante el control prenatal y son enviados a nivel terciario y por lo tanto, no llegan a la sala de partos del Centro Hospital Primitivo Iglesias. Tal es el caso de desproporción céfalo-pélvica e hipertensión arterial inducida por el $\mathrm{cm}$ barazo.

Conociendo aspectos específicos del embarazo en adolescentes como el parto prematuro es importante vigilar más de cerca a éste grupo de gestantes. Lo mismo sucede con el bajo peso al nacer lo cual merece un mayor énfasis en las consultas prenatales a cerca de una mayor ingesta proteico-calórica.

\section{BIBLIOGRAFIA}

\section{REFERENCIAS BIBLIOGRAFICAS}

1. SILVER, T.J. El embarazo en adolescentes en Estados Unidos de América, Salud Materno Infantil y Atencion Primaria en las Américas. Hechos y Tendencias. Publicación científica de la OPS 461. Washington, D.C., 1984, pp. 232-239.

2. OPS. Salud y Población. Boletín de la Oficina Sanitaria Panamericana. $\mathrm{XI}$, 1984; 97: $451-454$.

3. OMS. EI embarazo y el aborto en la adolescencia. Serie informes técnicos No. 583. Ginebra, Suiza, 1975; pp. 16-21.
4. ORGANIZACION PANAMERICANA DE LA SALUD. Las condiciones de salud en las Américas, 1977-1980. Publicación científica de la OPS 427 . Washington, D.C. 1982; pp. 23-34.

5. ZUCKERMAN, S. Barry. Adolescent pregnancy biobehavioral determinants of outcome. J. Pediat. 1984; 105: 857-863.

6. ROEMER, Ruth and PAXMAN, John $M$. Sex Education laws and policies. Studies in Family Planning. 1985; 16: 219-230.

7. RIZO, A. y PRADA H. Un Centro para la atención de la adolescente embaraza- 
da. Revista Col. Obst. Ginec. 1981, 32: 357-361.

8. RIZO, A. Y PRADA, H. La Fecundidad de la población de 10 a 19 años en Colombia. Rev. Col. Obst. Ginec. 1982; 33: 357-366.

9. HORION, Isabelle $y$ colaboradores. Birth weights among Infant born to adolescent and young adult woman. Am. J. Obstet. Gynecol. 1983; 146: 444-449.

10. CASSORLA S, Roosevelt. Suicidio y Adolescentes, factores sicosociales $y$ aspectos clínicos. Acta psiquiat. psicol. Amer. Lat. 1980; pp. 26-42.
11. ZUCKERMAN S, Barry y colaboradores, op. cit.

12. BELITZKY, Rubén y colaboradores. La adolescente embarazada. La salud del adolescente y el joven en las Américas. OPS/OMS. Publicación Científica No. 489. 1985. pp. 221-232.

13. SERIE PALTEX No. 7 OPS/OMS. Manual sobre el enfoque de riesgo en la atención materno-infantil. 1986, pp. 17.

14. GABRIELSON IW. et al. Suicide attemps in a population pregnant as teenagers. Amer. J. Public Health. 1970; 60: 22892301. 